\title{
AC 2009-836: EMPOWERING WOMEN AS LEADERS AND SCHOLARS: ADVANCE LEADERSHIP PROGRAMS AT A DOCTORAL STEM-DOMINANT UNIVERSITY
}

\section{Ane Johnson, Virginia Tech}

Ane Turner Johnson is the graduate assistant for AdvanceVT at Virginia Tech. Her research interests include higher education and development in Africa, undocumented student access to higher education, and gender issues in the academic sciences and engineering fields. She received her M.S. from George Mason University and her Ph.D. from Virginia Tech.

\section{Margaret Layne, Virginia Tech}

Peggy Layne, P.E., joined Virginia Tech in 2003 as director of the AdvanceVT program. Ms. Layne previously worked as a diversity consultant for the American Association of Engineering Societies and as director of the program on diversity in the engineering workforce at the National Academy of Engineering. Ms. Layne is a registered professional engineer with degrees in environmental and water resources engineering from Vanderbilt University and the University of North Carolina School of Public Health, and worked for 17 years as an environmental engineering consultant. She spent a year as an AAAS Congressional Fellow sponsored by the American Society of Civil Engineers and served as president of the Society of Women Engineers in 1996-97.

\section{Janis Terpenny, Virginia Tech}

Janis Terpenny is an Associate Professor in Mechanical Engineering and Engineering Education, and an affiliate faculty of Industrial \& Systems Engineering at Virginia Tech. She is Director of the Center for e-Design, a multi-university NSF I/UCRC center. Her research focuses on methods and representation schemes for early design process and on engineering design education. Dr. Terpenny was previously faculty at the University of Massachusetts and worked at General Electric (GE), including the completion of a two-year management program. She is a member of ASEE, ASME, IIE, and Alpha Pi Mu and is the Design Economics area editor for The Engineering Economist and the Associate Editor for the ASME Journal of Mechanical Design. She also serves on the AdvanceVT leadership team. 


\section{Empowering Women as Leaders: ADVANCE Leadership Programs at a Doctoral STEM-Dominant University}

STEM-dominant universities have been historically dominated by men and by traditions that, by their nature, have excluded women. ${ }^{1}$ For example, women make up only $6.9 \%$ of all full professors in engineering, nationwide. ${ }^{2}$ Women faculty members note disparities in numbers, salary, opportunities, resource allocation, and job satisfaction in general, and in the sciences and engineering more specifically. ${ }^{3}$ The lack of women in leadership positions has left many women junior faculty and graduate students feeling isolated and without the necessary support to build successful academic careers. ${ }^{4}$ Yet women faculty have indicated high levels of interest in leadership positions. ${ }^{5}$ With NSF ADVANCE support, Virginia Tech, a STEM-dominant university, has sought to empower women faculty to overcome the barriers to leadership.

This paper reports on how leadership programs focusing on women faculty can increase the representation of women in leadership roles across campus at a STEM-dominant institution. By providing multiple strategies to empower women faculty at varying stages of their careers, ADVANCE leadership programs sought to enhance their capabilities and productivity as technical and administrative leaders and as scholars. This was done, in part, by bringing women faculty together at many points during the project to reflect on issues of common concern and strategies for improvement, by encouraging individual women and men to use their positions and their work to address issues of concern to women in general, and to maximize the self and institutional reflection that sets the context for these strategies.

The purpose of this paper is to highlight the major elements of the Leadership Development Program implemented at Virginia Tech. First the authors provide a brief overview of the literature addressing women in academic leadership. Next descriptions of the university, ADVANCE program and leadership development initiative are summarized. Then university statistics and women faculty members' own words are used to depict the transformation from an institution with few women leaders to one that has invested in developing a culture of faculty development and success. Finally the authors will provide some conclusions regarding women's leadership programs and opportunities for improvement at the programmatic and institutional levels.

\section{Literature Review}

The literature on women in academic leadership tends to focus on three specific topics: pipeline ${ }^{6}$, mentoring ${ }^{7}$, and university climate ${ }^{8}$. Each of these issues acts, singularly or in combination, to support or prevent women pursuing leadership positions at universities in the United States.

\section{Leaky Pipeline}

It is often noted by scholars that sufficient numbers of women for the faculty pipeline exist. "The problem is that their status, once in the academy, is low." pursue academic careers and leadership positions because they are not encouraged to do so or 
lack proper role models. ${ }^{10}$ This intensifies as women persist through the pipeline and are confronted by university demographics that are distinctly homogenous. In fact, men continue to be the majority in the senior ranks at universities; and the more prestigious the institution, the wider the gap between men and women faculty in terms of numbers. ${ }^{11}$ Thus it appears that it is at the beginning and the center of the pipeline where the largest leaks occur and institutions lose out on qualified women.

\section{Mentoring}

The lack of mentoring is persistently identified as an obstacle to women attempting to move up faculty ranks. Research consistently implies that mentoring is highly valued among women in academe and should be used as a means to contribute to women's career success. ${ }^{12}$ Yet mentoring is erratically applied at universities and "even in the presence of female role models, the pressures against transcending traditional gender patterns are significant." ${ }^{13}$ Furthermore, the lack of high ranking women in academe to act as role models to female graduate students and younger faculty inculcates anxiety, increases attrition and ultimately prevents women from succeeding to leadership positions. ${ }^{14}$

\section{University Climate}

Beyond contending with ever decreasing resources, research institutions are being censured for the prevailing organizational paradigms on which they have been built; institutional values that have worked to keep outsiders, like women and minorities, out. ${ }^{15}$ Distinguished women faculty have reported that exclusion from the "old boy networks" at universities has impacted career success. ${ }^{16}$ Women may also have to cope with an environment where university policies are only grudgingly enforced (such as sexual harassment policies); policies that have a major impact on job satisfaction. ${ }^{17}$ Many women faculty members attribute their exit from academe to exclusion from decision making, ridicule and attention to sexuality in professional settings. ${ }^{18}$

These three concerns, either in concert or experienced individually, continue to have profound effects on women's entry into academe and their movement up faculty ranks into leadership positions. In the following paragraphs, the authors will describe Virginia Tech, an institution that faces these challenges, and its attempts to transform institutional culture and promote inclusivity towards women.

\section{Background}

\section{About the Institution}

Virginia Tech is a doctoral STEM-dominant institution (previously known as a Research I) located in Blacksburg, Virginia, a rural area in the southeastern United States. The university boasts over 30,000 students and over 104 doctoral programs. The university generated $\$ 366.9$ million for research programs in fiscal year 2007, ranking it 42nd in the nation. In the most recent fiscal year (2007-08), the university received more than 1,950 awards to conduct research. Today, nearly 775 sponsors fund more than 3,500 active projects. This research led to 33 patents 
and 17 licenses in 2007. As of fall of 2008, the institution had 1,371 tenured or tenure track faculty members, $22 \%$ of whom were female.

The largest cohort of women faculty currently resides in the College of Liberal Arts and Human Sciences, with women making up $44 \%$ of the total faculty in that college. This corresponds with national averages for those disciplines. In contrast, only $13 \%$ of the 315 tenure-track faculty in the College of Engineering are women, also comparable to national averages. The shortage of women at the professor level is acute, with women making up only $15 \%$ of full professors at the institution. The low number of women at the senior ranks means the pool of experienced women available for appointments to endowed professorships and chairs and important policy making committees is very limited. Compounding the problem, voluntary departure rates among women faculty members are twice that of men who choose to leave the institution.

Of the eight academic deans at the institution, two (Agriculture and Life Sciences and Liberal Arts and Human Sciences) are women. In addition, the Dean of Libraries is a woman. Nine $(36 \%)$ of the 25 associate deans in the academic colleges are women, plus one of two associate deans in the Graduate School (50\%). Only seven of the 62 (11\%) academic department heads are women; four of the seven women heads are in the College of Liberal Arts and Human Sciences. As of the fall of 2008, the institution has its first female department head in the College of Engineering. The College of Business also gained a female department head in fall 2008 in the Department of Management. Women are also scarce in senior-level leadership positions at the university. The most senior positions (president, provost, and vice president) are held primarily by men; three of $14(21 \%)$ executive administrators are women. Three women $(21 \%)$ are directors of university-level research centers.

\section{ADVANCE}

In 2003, Virginia Tech received a five-year National Science Foundation ADVANCE Institutional Transformation grant. The NSF ADVANCE program seeks to "develop systemic approaches to increase the representation and ADVANCEment of women in academic science and engineering careers, thereby contributing to the development of a more diverse science and engineering workforce." ${ }^{19}$ Implementation of grant programs and activities is housed in the Office of the Provost. The institution's ADVANCE program seeks to contribute to the development of an academic science and engineering workforce that includes the full participation of women at all levels of faculty and academic leadership, particularly at the senior academic ranks, through the transformation of institutional practices, policies, climate, and culture.

The program addresses institutional transformation in a variety of ways. The program funded Ph.D. and post-doctoral fellowships for women researchers. Seminars and workshops target women graduate students and faculty members and address skill attainment in writing grant proposals, interviewing for academic jobs, negotiating academic job offers, and obtaining leadership roles within the university. The project also assists university deans, department heads and administrators to identify and address unconscious bias, to institute diverse hiring practices, and to develop mentoring programs for faculty. The program supports faculty recruitment though 
a visiting scholar program and collaboration with the Office for Equal Opportunity to create a database and attend recruitment fairs for prospective minority faculty.

Leadership Development Programs

The institution's ADVANCE program sponsors a series of workshops and events to build specific leadership skills, including communication skills, resolving conflict, negotiation, power and influence, and time management. These workshops utilize both on campus experts and outside consultants. Leadership seminars for senior women faculty give them the opportunity to interact with current leaders across the university and outside the institution, including department heads, associate deans, deans, research center directors, vice-presidents, and provosts. In addition to seminars and workshops open to all faculty, the institution's ADVANCE Leadership Development Program selected three cohorts of women from across the university who demonstrated an interest in and potential to assume leadership roles for a year of individualized coaching and skill development. Working with a coach, each woman received a "360 degree" evaluation that solicited input from her superiors (department head), peers, and subordinates (graduate students, admin support, and/or technicians) and based on this input created a development plan to capitalize on strengths and address development needs. Regular follow up with the coach encouraged completion of the plan. Leadership Fellowships were awarded to six women faculty members to complete self-designed leadership development experiences. Fellowship recipients received course buy-outs to develop research centers, establish graduate programs, organize research symposiums, and work with leadership in their departments and colleges to develop programs and policies that improve departmental climate as well as to review curriculum.

\section{Evaluation Outcomes}

Program evaluations were carried out at intervals over the past five years of the grant. Workshop and seminar participants were invited to provide feedback using brief paper and online surveys. In addition, interviews were conducted with female faculty members (across disciplines) who completed the Leadership Development Program and received Leadership Fellowships. Finally, results from two university-wide faculty surveys will be presented to demonstrate changing faculty attitudes toward leadership and university climate.

Leadership Development Event Evaluations

Since 2005, the institution's ADVANCE program has provided leadership development events for faculty members covering a wide array of topics related to the roles, responsibilities, and rewards of being an academic leader and providing an opportunity to network with university leaders across colleges and departments. In year five, the program conducted a cumulative evaluation of the leadership development seminars. Participants were asked to complete a survey to evaluate the usefulness and relevance of the faculty leadership events. A total of 121 individuals attended one or more of these seminars, and 42 participants responded to the survey. 
Many respondents noted the importance of networking with other female faculty members and administrators across the institution and the potential for such opportunities to bridge gaps between faculty and administration.

These [events] have been an effective offering and good networking. The more faculty can understand the thinking of university leaders, the more engaged they can be as well as supportive.

The events were successful in educating faculty on various aspects of academic leadership and changing faculty perception of leadership and leaders and the skills needed to be successful.

Leadership positions are much more complex than they appear to be from the outside - it takes much more than just people skills to succeed.

Forty-five percent of respondents indicated that they were potentially interested in a leadership position at the university and 57\% noted that the leadership events were an effective approach to leadership development. Yet many responses reveal that women faculty at the institution continue to be ambivalent about entering into leadership positions.

Unfortunately, [the leadership events] make the realities of the pressures to advance crystal clear. It is disheartening that we ... are continually forced to raise the bar on faculty promotion based on research due to continued budget cuts. This cuts out a lot of REALLY great leaders who are currently mid-career, but not interested in clawing their way to the next promotion level. It is also impressive, yet intimidating, what a serious time commitment these administrative positions require.

Much of this ambivalence appears to originate in balancing the demands of leadership positions with that of research productivity.

[The leadership event] emphasized how difficult it is to be in a leadership position and continue in research productivity.

When asked what other leadership topics attendees would like to see addressed by the leadership events, many faculty members demonstrated concern over prevailing university culture and the necessary skills to manage it. One participant noted that that she was interested in "Get[ting] around the good old boy network and/or fend off cronyism and insider deal making." Yet another participant desired insight into relationships among administrators, particularly "why there is apparently such a gulf in understanding between department heads/center directors and the deans/provost?"

Leadership Development Program Interviews

In 2008, the program conducted interviews with women faculty who had participated in the Leadership Development Program (LDP) and Leadership Fellowships. Nineteen phone interviews (of the 23 women faculty who participated overall) were conducted with faculty on expected and actual outcomes of their participation. The interview protocol asked faculty 
members about their motivation to participate in the program, the skills they hoped to acquire and to identify tangible outcomes attributable to their participation.

While all participants indicated that their leadership skills and confidence to pursue leadership opportunities increased, only a few of the participants expressed a desire in the near future to pursue leadership positions.

I shadowed a provost office administrator... and this experience helped to know, precisely, that I am not interested in taking on a leadership position in that capacity. He was so harried, and it was difficult to get to spend time with him. I just don't think I'm ready, or ever will be, for that kind of pace.

Participants felt that the program helped them to attain greater success in their current positions and to participate more fully in department leadership opportunities.

I accepted a department head's position and without those two experiences [LDP and a national leadership program] I would have never considered it...

I've actually submitted materials to be a full professor this year, but who knows...I've transitioned from co-director to director of an NSF center...I don't know if I would attribute it entirely to the program, but it's definitely due to better leadership skills.

Yet, consistent with data from the leadership event evaluations, most participants articulated the belief that administrative roles required much more time and effort than originally expected. Furthermore, such positions take away from activities traditionally associated with being a faculty member, such as research and teaching.

While the ADVANCE programs focused primarily on preparing mid-career faculty to move into leadership roles, one participant mentioned her efforts to address the leaky pipeline for female graduate students by including them in leadership events and the need for more opportunities for female graduate students to network and meet other successful women faculty.

The point when you bring a visitor [to campus] you usually talk about your research, it's very hard to [include] my students, I have two female graduate students and one undergrad and they are always involved in the interaction with the external distinguished faculty members somehow. It is a very good idea and always a networking activity is very good, I wish we could involve graduate students more, usually in the science area it is very hard to get them out of the lab.

2005 \& 2008 Faculty Work-Life Survey

In the spring of 2005, the ADVANCE program in conjunction with the institution's Survey Research Center distributed a survey, to which 219 tenured and tenure track women and 599 tenured and tenure track men responded. In response to the statement "There are too few women and minorities in leadership positions at [the institution]," $89.7 \%$ of women and $59.8 \%$ of men agreed. In contrast, when responding to the statement "I aspire to a leadership position at [the 
institution] beyond my current position," $46.6 \%$ of women agreed, whereas $30.5 \%$ of men agreed. However, $47.9 \%$ of men but only $29.1 \%$ of women agreed that it is possible to hold a leadership position at the institution while balancing work and personal responsibilities. Finally, when asked whether they were interested in opportunities to develop leadership skills, $77.3 \%$ of the women responded in the affirmative compared with only $55.4 \%$ of men.

A follow up survey was conducted in fall 2008, and received responses from 223 female and 477 male tenured and tenure-track faculty, a response rate of $67.8 \%$ for the women and $47.7 \%$ for the men. Of the respondents, $76.8 \%$ of the women compared with $43.8 \%$ of the men agreed that "There are too few women in leadership positions at [the institution]". (Note that the wording of this question was slightly different in 2008 , separating women and underrepresented minorities into two questions.) In contrast to 2005, about the same percentage of men and women aspired to leadership roles beyond their current position (34\% of women and 35\% of men). Compared with 2005, about the same percentage of women (26\% in 2008 vs. $29 \%$ in 2005) but a smaller percentage of men (39\% vs. $48 \%$ ) agree that it is possible to hold a leadership position while balancing work and personal responsibilities. A slightly smaller percentage of women (63\% in 2008 vs. $77 \%$ in 2005) but the same percentage of men (55\%) were interested in developing their leadership skills.

\section{Discussion and Implications}

Findings from these evaluations suggest that while the university climate may be warming towards women pursuing leadership positions, women themselves continue to exhibit conflicting attitudes towards leadership. The main issue elicited from the data appears to be concern over the amount of work involved and the corresponding time commitment required in taking on leadership positions as a faculty member. Women with young families who are still at the associate professor level are particularly hesitant to take on formal leadership roles given the importance of research output for achieving promotion to professor. Many of the participants in the Leadership Development Program were at the associate professor level, and do not plan to pursue formal academic leadership positions (e.g., department head, associate dean) until after their promotion to professor. Nevertheless, participation in leadership development activities offered by the ADVANCE program increased awareness of the challenges and rewards of academic leadership, provided opportunities to interact with current leaders, and facilitated development of leadership skills.

STEM-dominant institutions interested in diversifying their pool of potential leaders must not only provide resources to help individuals prepare for leadership roles (skill development opportunities, mentoring, and access to role models), they must also examine their expectations of leaders and structure of leadership positions in order to attract women into leadership. Additionally, policies that facilitate work-life balance for faculty members may also play a role in filling the pipeline of qualified women for leadership positions. Policies such as modified duties, stop-the-clock, and dual career assistance may help to retain women faculty members and increase the percentage of women in associate and full professorships. This increase may also provide more junior women with effective role models and mentors and repair the leak at the beginning of the pipeline. 
Regarding university climate, future research should be undertaken to delve more fully into the success of the ADVANCE grants in transforming universities, and the subsequent way these efforts impact the way leadership is defined at those institutions. Longitudinal research could be undertaken to measure leadership paradigms (expectations for how leaders should behave) of faculty members at the beginning and end of an ADVANCE grant. For institutions concluding their ADVANCE grants, data collection using post measures only could be employed and compared to peers or existing data on leadership paradigms.

\section{Conclusion}

The current research suggests that leadership awareness and skill development programs and events are only part of the solution to the triumvirate of leaky pipeline, mentoring and university climate that inhibits women from seeking leadership positions. Evaluations of these activities indicate that such programs alone are not effective in convincing women faculty members to undertake leadership positions. These results have not dissuaded Virginia Tech from continuing to prepare women faculty for leadership roles. In fact, we are further encouraged as these activities were effective in changing women's perception of leaders and their own abilities and skills as leaders. This self-awareness will no doubt put more women faculty on the path to success in leadership.

\footnotetext{
1 Fox, M. F. (1996). "Women, academia, and careers in science and engineering" in The Equity Equation: Fostering the Advancement of Women in the Sciences, Mathematics, and Engineering. (Eds.) Davis, C., A. Ginorio, C. Hollenshead, B. Lazarus, P. Rayman \& Associates. San Francisco, CA: Jossey-Bass.

${ }^{2}$ Gibbons, M. (2007). Engineering by the Numbers. Association for Engineering Education Report. Washington, DC.

${ }^{3}$ The Collaborative of Academic Careers in Higher Education. (2007). COACHE Highlights Report December 2008. Cambridge, MA: Harvard University.

4 Etzkowitz, H., Kemelgor, C., Neuschatz, M. \& Uzzi, B. (1994). "Barriers to women's participation in academic science and engineering” in Who Will Do Science? (Eds.) Pearson, W. \& A. Fetcher. Baltimore, MD: Johns Hopkins University Press.

5 Internal Institution Report (2005)

${ }^{6}$ Handelsman, J., Cantor, N., Carnes, M., Denton, D., Fine, E., Grosz, B., Hinshaw, V., Marrett, C., Rosser, S., Shalala, D. \& Sheridan, J. (2005 August). More women in science. Science Magazine 309. 1190-1191.

Trower, C. \& Chait, R. (2002 April). Faculty diversity: Too little for too long. Harvard Magazine.

Stewart, A. Malley, J. \& LaVaque-Manty, D. (2007). Transforming Science and Engineering: Advancing Academic Women. Ann Arbor, MI: University of Michigan Press.

7 Etzkowitz, et. al. (1994)

Gibson, S. (2004). Being mentored: The experience of women faculty. Journal of Career Development 30(3). 173188.

Stewart, A. Malley, J. \& LaVaque-Manty, D. (2007). Transforming Science and Engineering: Advancing Academic Women. Ann Arbor, MI: University of Michigan Press.

Yedida, M. \& Bickel, J. (2001). Why aren't there more women leaders in academic medicine? The view of clinical department chairs. Academic Medicine 76(5). 462.

8 Cole, J. (1993). Balancing acts: Dilemmas of choice facing research universities. Daedalus 122(4). 1-36.

Handelsman, J., Cantor, N., Carnes, M., Denton, D., Fine, E., Grosz, B., Hinshaw, V., Marrett, C., Rosser, S., Shalala, D. \& Sheridan, J. (2005 August). More women in science. Science Magazine 309. 1190-1191.
} 
Ropers-Huilman, B. (2000). Aren't you satisfied yet? Women faculty members' interpretations of their academic work. New Directions for Institutional Research (105). 21-32.

Stewart, et. al. (2001).

Rosser, S. (2004). The Science Glass Ceiling: Academic Women Scientists and the Struggle to Succeed. New York, NY: Routledge.

${ }^{9}$ Trower \& Chait (2002) p. 34.

${ }^{10}$ Handelsman, et. al. (2005)

${ }^{11}$ Trower \& Chait (2002)

${ }^{12}$ Gibson (2004); Rosser (2004)

${ }^{13}$ Yedida \& Bickel (2001) p. 462.

${ }^{14}$ Etzkowitz, et. al. (1994)

${ }^{15}$ Cole (1993)

${ }^{16}$ Rosser (2004)

${ }^{17}$ Ropers-Huilman (2000)

${ }^{18}$ Handelsman, et. al. (2005)

${ }^{19} \mathrm{http} / / /$ www.nsf.gov/funding/pgm_summ.jsp?pims_id=5383 